\title{
Products of Lower Densities
}

\author{
N. D. Macheras and W. Strauss
}

\begin{abstract}
We prove the existence of product-lower densities for products of arbitrary families of (possibly incomplete) probability spaces, thus generalizing the corresponding results for finite products. The proofs are based on permanence results for lower densities under projective limits of probability spaces. Relying on work of Grekas we discuss in particular Haar measures on compact groups.
\end{abstract}

Keywords: Lower densities, product measures, Haar measures

AMS subject classification: Primary 28 A 51, sccondary 60 B 05,60 B 15

\section{Introduction}

In [10] and [11] the existence of the so-called product lifting, i.e. of a lifting compatible with the product, has been established for the completed product of an arbitrary family of complete probability spaces. This raises the question what the position is for noncomplete probability spaces. Since it is known that for non-complete probability spaces liftings, in general, do not exist (see [15]) we replace liftings by lower densities whose existence is garanteed by [4] for arbitrary probability spaces.

In the main theorem of this paper (Theorem 2 of Section 3) we establish the existence of a product - lower density for arbitrary families of (possibly incomplete) probability spaces where we use the following terminology:

Given a family $\left(\left(\Omega_{i}, \Sigma_{i}, \mu_{i}\right)\right)_{i \in I}$ of probability spaces and another probability space $(\Omega, \Sigma, \mu)$ satisfying

$$
\Omega=\prod_{i \in I} \Omega_{i}, \quad \Sigma \supseteq \bigotimes_{i \in I} \Sigma_{i}, \quad \mu \mid \bigotimes_{i \in I} \Sigma_{i}=\bigotimes_{i \in I} \mu_{i}
$$

we then call a lower density $\pi$ for $\mu$ a product - lower density of the lower densities $\varphi_{i}$ for $\mu_{i}(i \in I)$ and we write $\pi=\otimes_{i \in I} \varphi_{i}$ if the equation

(P) $\pi\left(\left[A_{i_{1}}, \ldots, A_{i_{n}}\right]\right)=\left[\varphi_{i_{1}}\left(A_{i_{1}}\right), \ldots, \varphi_{i_{n}}\left(A_{i_{n}}\right)\right]$

N. D. Macheras: Univ. Piraeus, Dep. Statistics, 80 Karaoli and Dimitriou Street, 18534 Piracus, Greece

W. Strauss: Univ. Stuttgart, Math. Inst. A, PF 8011 40, D - 70511 Stuttgart

ISSN 0232-2064 / \$2.50 (C) Heldermann Verlag Berlin 
holds true for all $i_{1}, \ldots, i_{n} \in I$ and all $A_{i_{k}} \in \Sigma_{i_{k}}(k=1, \ldots, n)$ where $\left[A_{i_{1}}, \ldots, A_{i_{n}}\right]$ denotes the cylinder set $\prod_{i \in I} B_{i}$ for $B_{i_{k}}=A_{i_{k}} \quad(k=1, \ldots, n)$ and $B_{i}=\Omega_{i}$ if $i \in$ $I \backslash\left\{i_{1}, \ldots, i_{n}\right\}$.

The proof of this basic existence result relies on the permanence theorem for lower densities under projective limits of Section 2 and is in so far quite different from the methods taken in [11] for the proof of the product-lifting theorem.

Based on work of Grekas [5] we construct for the Haar measure on a compact group a lower density which is a product of lower densities for Baire (=Borel) probability measures supported by compact Lie (hence metrizable) groups (see Theorem 3 in Section 3).

\section{Notations}

For a given probability space $(\Omega, \Sigma, \mu)$ a set $N \in \Sigma$ with $\mu(N)=0$ is called a $\mu$-null set, and for sets $A, B \in \Sigma$ we write $A=B \mu$-a.e. if $A \triangle B$, the symmetric difference of $A$ and $B$, is a $\mu$-null set. The (Carathéodory) completion of $(\Omega, \Sigma, \mu)$ will be denoted by $\left(\Omega, \Sigma_{\mu}, \hat{\mu}\right)$. When there is no danger of confusion we write $\hat{\Sigma}$ in place of $\Sigma_{\mu}$. We use the notion of (lower) density (resp. lifting) in the sense of 17: Chapter III, Section $1 /$ Def. 4 (resp. Def. 3)] and for any probability space $(\Omega, \Sigma, \mu)$ we denote by $\vartheta(\Sigma, \mu)$ or just by $\vartheta(\mu)$ (resp. by $\Lambda(\Sigma, \mu)$ or just by $\Lambda(\mu)$ ) the system of all (lower) densities (resp. liftings).

We denote by

$$
\left(\prod_{i \in I} \Omega_{i}, \bigotimes_{i \in I} \Sigma_{i}, \bigotimes_{i \in I} \mu_{i}\right) \quad \text { or } \quad \bigotimes_{i \in I}\left(\Omega_{i}, \Sigma_{i}, \mu_{i}\right)
$$

the product probability space of the probability spaces $\left(\Omega_{i}, \Sigma_{i}, \mu_{i}\right) \quad(i \in I)$ and by

$$
\left(\prod_{i \in I} \Omega_{i}, \widehat{\bigotimes_{i \in I}} \Sigma_{i}, \widehat{\bigotimes_{i \in I}} \mu_{i}\right) \quad \text { or } \quad \widehat{\bigotimes_{i \in I}}\left(\Omega_{i}, \Sigma_{i}, \mu_{i}\right)
$$

its (Carathéodory) completion.

We use the notions of projective systems and projective limits of probability spaces in the sense of [14]. For a projective system $\left(\Omega_{\alpha}, \Sigma_{\alpha}, \mu_{\alpha}, f_{\alpha \beta}, I\right)$ of probability spaces a family of (lower) densities $\varphi_{\alpha} \in \vartheta\left(\mu_{\alpha}\right)(\alpha \in I)$ is called self-consistent if

$$
\varphi_{\beta} \circ f_{\alpha \beta}^{-1}=f_{\alpha \beta}^{-1} \circ \varphi_{\alpha} \text { for all } \alpha, \beta \in I \text { with } \alpha \leq \beta
$$

If $\left(\Omega, \Sigma, \mu,\left(f_{\alpha}\right)_{\alpha \in I}\right)$ is the projective limit of the above projective system a (lower) density $\varphi \in \vartheta(\mu)$ is called a projective limit of the self-consistent family $\left(\varphi_{\alpha}\right)_{\alpha \in I}$ if

$$
\varphi \circ f_{\alpha}^{-1}=\dot{f}_{\alpha}^{-1} \circ \varphi_{\alpha} \quad \text { for all } \alpha \in I,
$$

in symbols $\varphi=\operatorname{proj}_{\alpha \in I} \lim \varphi_{\alpha}$. 
We suppose throughout that all the canonical projections $f_{\alpha}$ from $\Omega$ onto $\Omega_{\alpha}$ are surjective. All the densities used in this paper are lower densities so that for simplicity we will use the word "density" instead of "lower density".

Throughout, $X$ denotes a completely regular Hausdorff topological space, and $C_{\mathrm{b}}(X)$ is the space of all bounded continuous real-valued functions on $X$. The $\sigma$-field of Borel sets $\mathcal{B}(X)$ (respectively Baire sets $\mathcal{B}_{0}(X)$ ) over $X$ is the one generated by the open subsets of $X$ (respectively by the zero sets of $X$ ). For a probability measure $\mu$ on $\hat{\mathcal{B}}(X)$ a lifting $\rho \in \Lambda(\mu)$ is called strong if $G \subseteq \rho(G)$ for any open subset $G \subset X$.

Let $X_{1}$ and $X_{2}$ be two topological spaces. A mapping $g: X_{1} \rightarrow X_{2}$ is called Baire measurable if it is $\left(\mathcal{B}_{0}\left(X_{1}\right)-\mathcal{B}_{0}\left(X_{2}\right)\right)$-measurable. A Baire measurable bijection $g$ is said to be a Baire isomorphism if $g^{-1}$ is also Baire measurable.

For unexplained notions of measure theory or of topological groups we refer to Halmos [6] or to Montgomery and Zippin [13], respectively.

\section{A permanence result}

The main theorem of this section is the following permanence result for densities under projective limits. It is basic for the main theorem of the next section, but may also be of some interest in its own.

Theorem 1. Let $\left(\Omega, \Sigma, \mu,\left(f_{\alpha}\right)_{\alpha \in I}\right)$ be the projective limit of a projective system $\left(\Omega_{\alpha}, \Sigma_{\alpha}, \mu_{\alpha}, f_{\alpha \beta}, I\right)$ of probability spaces. Suppose that $\left(\varphi_{\alpha}\right)_{\alpha \in I}$ is a self-consistent family of densities $\varphi_{\alpha} \in \vartheta\left(\mu_{\alpha}\right)$. Then there exists a $\varphi \in \vartheta(\mu)$ which is a projective limit of $\left(\varphi_{\alpha}\right)_{\alpha \in I}$.

Proof. For every $\alpha \in I$ we set $\Sigma_{\alpha}^{*}=f_{\alpha}^{-1}\left(\Sigma_{\alpha}\right)$ and $\mu_{\alpha}^{*}=\mu \mid \Sigma_{\alpha}^{*}$ and define a density $\varphi_{\alpha}^{*}$ for $\left(\Omega, \Sigma_{\alpha}^{*}, \mu_{\alpha}^{*}\right)$ by

$$
\varphi_{\alpha}^{*}\left(f_{\alpha}^{-1}(A)\right)=f_{\alpha}^{-1}\left(\varphi_{\alpha}(A)\right) \quad \text { for every } \quad A \in \Sigma_{\alpha}
$$

For all $\alpha, \beta \in I$ with $\alpha \leq \beta$ we have $\Sigma_{\alpha}^{*} \subseteq \Sigma_{\beta}^{*}$ and $\varphi_{\beta}^{*} \mid \Sigma_{\alpha}^{*}=\varphi_{\alpha}^{*}$. Indeed, for every $A \in \Sigma_{\alpha}$

$$
f_{\alpha}^{-1}(A)=f_{\beta}^{-1}\left(f_{\alpha \beta}^{-1}(A)\right) \in \Sigma_{\beta}^{*}
$$

because $f_{\alpha \beta}^{-1}(A) \in \Sigma_{\beta}$ and so

$$
\begin{aligned}
\varphi_{\beta}^{*}\left(f_{\alpha}^{-1}(A)\right) & =f_{\beta}^{-1}\left(\varphi_{\beta}\left(f_{\alpha \beta}^{-1}(A)\right)\right)=f_{\beta}^{-1}\left(f_{\alpha \beta}^{-1}\left(\varphi_{\alpha}(A)\right)\right) \\
& =f_{\alpha}^{-1}\left(\varphi_{\alpha}(A)\right)=\varphi_{\alpha}^{*}\left(f_{\alpha}^{-1}(A)\right)
\end{aligned}
$$

We distinguish two cases.

(i) There is a countable cofinal subset $J \subset I$. Then $\Sigma$ is the $\sigma$-algebra generated by $\cup_{\alpha \in J} \Sigma_{\alpha}^{*}$ and by [3: Lemma 1] there exists $\varphi \in \vartheta(\mu)$ such that $\varphi \mid \Sigma_{\alpha}^{*}=\varphi_{\alpha}^{*}$ for every $\alpha \in I$, i.e. $\varphi$ is a projective limit of $\left(\varphi_{\alpha}\right)_{\alpha \in I}$.

(ii) There is no countable cofinal subset of $I$. Then $\Sigma=\cup_{\alpha \in I} \Sigma_{\alpha}^{*}$ and the desired density $\varphi$ for $\mu$ is given by $\varphi(A)=\varphi_{\alpha}^{*}(A)$ if $A \in \Sigma_{\alpha}^{*}$ and $\alpha \in I$ 
There are projective systems of probability spaces for which self-consistent families of densities arise naturally (see [9: Remark 2.2]). The next proposition exhibits another such class of spaces in a very general situation. As a preparation we need the following lemma which is an analogue of [8: Lemma 2.1].

Lemma 1. Let be given two probability spaces $(X, \Sigma, \mu)$ and $(Y, \Theta, \nu)$ and $a(\Sigma-\Theta)$ measurable map $f: X \rightarrow Y$ satisfying $\mu\left(f^{-1}(B)\right)=\nu(B)$ for all $B \in \Theta$. Then for any $\theta \in \vartheta(\nu)$ there exists a $\varphi \in \vartheta(\mu)$ such that $\varphi \circ f^{-1}=f^{-1} \circ \theta$.

Proof. Let $\mathcal{B}$ be the $\sigma$-subalgebra of $\Sigma$ generated by $f^{-1}(\Theta) \cup u$ for $u=\{N \in \Sigma$ : $\mu(N)=0\}$. Define a density $\varphi_{0}$ for $\bar{\mu}:=\mu \mid \mathcal{B}$ by $\varphi_{0}(A)=f^{-1}(\theta(B))$ for $A \in \mathcal{B}$ and $B \in \Theta$ with $A=f^{-1}(B) \bar{\mu}$-a.e. Now using arguments similar those in Traynor's proof (see [16: Theorem 6]) of the theorem of D. Maharam [12] we conclude that $\varphi_{0}$ has an extension $\varphi \in \vartheta(\mu)$. We have only to apply Lemmas 1 and 2 of [4] instead of Theorem 4 and Lemma 5 of [16], respectively, and to consider the set

$$
\mathcal{L}=\{(\mathcal{A}, \varphi) \mid \mathcal{A} \text { a-algebra in } X \text { with } \mathcal{B} \subseteq \mathcal{A} \subseteq \Sigma \text { and } \varphi \in \vartheta(\mu \mid \mathcal{A})\}
$$

instead of the set $\mathcal{H}$ used in [16: Theorem 6]. The standard argument using Zorn's lemma completes the proof

In the next proposition we shall identify a cardinal $\kappa$ with the initial ordinal of that cardinal.

Proposition 1. For an at least countably infinite cardinal $\kappa$ let be given a projective system $\left(X_{\alpha}, \Sigma_{\alpha}, \mu_{\alpha}, f_{\alpha \beta}, \kappa\right)$ of probability spaces with projective limit $\left(X, \Sigma, \mu,\left(f_{\alpha}\right)_{\alpha \in \kappa}\right)$ such that, for any limit ordinal $\lambda<\kappa$,

$$
\left(X_{\lambda}, \Sigma_{\lambda}, \mu_{\lambda}\right)=\operatorname{proj}_{\alpha<\lambda} \lim \left(X_{\alpha}, \Sigma_{\alpha}, \mu_{\alpha}\right)
$$

holds true. Then there exists a self-consistent family $\left(\varphi_{\alpha}\right)_{\alpha \in \kappa}$ of densities $\varphi_{\alpha} \in \vartheta\left(\mu_{\alpha}\right)$ for $\alpha \in \kappa$ and $a \varphi \in \vartheta(\mu)$ which is a projective limit of the family $\left(\varphi_{\alpha}\right)_{\alpha \in \kappa}$.

Proof. We construct a self-consistent family $\left(\varphi_{\alpha}\right)_{\alpha \in \kappa}$ of densities by induction on $\alpha \leq \kappa$ : For $\alpha=0$ we choose $\varphi_{0} \in \vartheta\left(\mu_{0}\right)$ by [4: Theorem 1]. If $\alpha=\beta+1<\kappa$ and $\varphi_{\beta} \in \vartheta\left(\mu_{\beta}\right)$ is known, we choose by Lemma 1 a $\varphi_{\beta+1} \in \vartheta\left(\mu_{\beta+1}\right)$ such that

$$
\varphi_{\alpha} \circ f_{\beta \alpha}^{-1}=f_{\beta \alpha}^{-1} \circ \varphi_{\beta} \text {. }
$$

Since $\varphi_{\beta} \circ f_{\delta \beta}^{-1}=f_{\delta \beta}^{-1} \circ \varphi_{\delta}$ for all $\delta<\beta$ we get $\varphi_{\alpha} \circ f_{\delta \alpha}^{-1}=f_{\delta \alpha}^{-1} \circ \varphi_{\delta}$ from (1), i.e. $\left(\varphi_{\delta}\right)_{\delta \leq \alpha}$ is self-consistent. For a limit ordinal $\alpha \leq \kappa$ we find by Theorem 1 a $\varphi_{\alpha} \in \vartheta\left(\mu_{\alpha}\right)$ such that $\varphi_{\alpha}=\operatorname{proj}_{\beta<\alpha} \lim \varphi_{\beta}$, i.c. $\left(\varphi_{\beta}\right)_{\beta \leq \alpha}$ is self-consistent. Thus the induction can be pushed through to obtain a self-consistent family $\left(\varphi_{\alpha}\right)_{\alpha \leq \kappa}$. In particular $\left(\varphi_{\alpha}\right)_{\alpha<\kappa}$ is self-consistent and $\varphi=\operatorname{proj}_{\alpha<\kappa} \varphi_{\alpha}$ if $\varphi:=\varphi_{\kappa}$

Remark 1. From the proof of the above proposition it follows that one of the $\varphi_{\alpha}$ can be pre-assigned or even more of them, i.e. under the assumptions of Proposition 1 , if $\left(\varphi_{\alpha}\right)_{\alpha \leq \beta} \quad(\beta<\kappa)$ is a pre-assigned self-consistent family of densities $\varphi_{\alpha} \in \vartheta^{\prime}\left(\mu_{\alpha}\right)$, then there exist a self-consistent family $\left(\varphi_{\gamma}\right)_{\beta<\gamma<\kappa}$ of $\varphi_{\gamma} \in \vartheta\left(\mu_{\gamma}\right)$ and a $\varphi \in \vartheta(\mu)$ such that $\varphi$ is a projective limit of $\left(\varphi_{\alpha}\right)_{\alpha<\kappa}$. In particular for $\beta=0$ we have that for a given $\varphi_{0} \in \vartheta\left(\mu_{0}\right)$ there exist a self-consistent family $\left(\varphi_{\alpha}\right)_{0<\alpha<\kappa}$ of $\varphi_{\alpha} \in \vartheta\left(\mu_{\alpha}\right)$ and a $\varphi \in \vartheta(\mu)$ such that $\varphi$ is a projective limit of $\left(\varphi_{\alpha}\right)_{\alpha<\kappa}$. 


\section{The product-density theorem}

The next theorem is the "product-density theorem" which generalizes Theorem 3 from [10: Section 2].

Theorem 2. Let be given probability spaces $(\Omega, \Sigma, \mu)$ and $\left(\Omega_{i}, \Sigma_{i}, \mu_{i}\right)$ for $i \in I, I$ a non-empty index set, such that

$$
\Omega=\prod \Omega_{i}, \quad \bigotimes_{i \in I} \Sigma_{i} \subseteq \Sigma, \quad \mu \mid \bigotimes_{i \in I} \Sigma_{i}=\bigotimes_{i \in I} \mu_{i}
$$

Fix on an $i_{0} \in I$. Then for any $\varphi_{i_{0}} \in \vartheta\left(\mu_{i_{0}}\right)$ there exist $\varphi_{i} \in \vartheta\left(\mu_{i}\right)$ for $i \in I \backslash\left\{i_{0}\right\}$ and $\pi \in \vartheta(\mu)$ such that $\pi=\otimes_{i \in I} \varphi_{i}$.

Proof. Let $\mathcal{C}$ be the family of all triples $\left(M,\left(\rho_{i}\right)_{i \in M}, \rho\right)$ where $M$ is a subset of $I$ with $i_{0} \in M, \rho_{i_{0}}=\varphi_{i_{0}}, \rho_{i} \in \vartheta\left(\mu_{i}\right)$ for every $i \in M, \rho \in \vartheta\left(\otimes_{i \in M} \mu_{i}\right)$ and $\rho=\otimes_{i} \in M \rho_{i}$. Define an order relation $\leq$ on $\mathcal{C}$ by

$$
\begin{aligned}
\left(M,\left(\rho_{i}\right)_{i \in M}, \rho\right) & \leq\left(N,\left(\tau_{i}\right)_{i \in N}, \tau\right) \\
& \Longleftrightarrow M \subseteq N, \tau_{i}=\rho_{i} \text { for all } i \in M, \tau \circ \pi_{M N}^{-1}=\pi_{M N}^{-1} \circ \rho
\end{aligned}
$$

where $\pi_{M N}: \prod_{i \in N} \Omega_{i} \longrightarrow \prod_{i \in M} \Omega_{i}$ denotes the natural projection.

If $\left(M_{\alpha},\left(\rho_{i}^{\alpha}\right)_{i \in M_{\alpha}}, \rho_{\alpha}\right)_{\alpha \in A}$ is a totally ordered subfamily of $\mathcal{C}$, we set $M=\cup_{\alpha \in A} M_{\alpha}$ and $\rho_{i}=\rho_{i}^{\alpha}$ for every $i \in M_{\alpha}$ and $\alpha \in A$. Further, identifying the projective limit of

$$
\left(\prod_{i \in M_{a}} \Omega_{i}, \bigotimes_{i \in M_{a}} \Sigma_{i}, \bigotimes_{i \in M_{a}} \mu_{i}, \pi_{M_{a} M_{\rho}}, A\right)
$$

with

$$
\left(\prod_{i \in M} \Omega_{i}, \bigotimes_{i \in M} \Sigma_{i}, \bigotimes_{i \in M} \mu_{i},\left(\pi_{M_{0} M}\right)_{\alpha \in A}\right)
$$

by Theorem 1 of Section 1 we find $\rho \in \vartheta\left(\otimes_{i \in M \mu_{i}}\right)$ such that $\rho \circ \pi_{M_{\alpha} M}^{-1}=\pi_{M_{\alpha} M^{\circ}}^{-1} \rho_{\alpha}$ for every $\alpha \in A$. It is clear that $\left(M,\left(\rho_{i}\right)_{i \in M}, \rho\right)$ is an upper bound of $\left(M_{\alpha},\left(\rho_{i}^{\alpha}\right)_{i \in M_{\alpha}}, \rho_{\alpha}\right)_{\alpha \in A}$ in $\mathcal{C}$. Thus $\mathcal{C}$ is inductively ordered and by Zorn's lemma there exists a maximal element $\left(M,\left(\varphi_{i}\right)_{i \in M}, \varphi\right)$ of $\mathcal{C}$.

We claim that $M=I$. Indeed, if $M$ were a proper subset of $I$ and $j \in I \backslash M$, by [10: Section 2/Theorem 3] we could choose $\varphi_{j} \in \vartheta\left(\mu_{j}\right)$ and $\psi \in \vartheta\left(\otimes_{i \in M \cup\{j\}} \mu_{i}\right)$ such that $\psi=\varphi \otimes \varphi_{j}$. But then $\left(M \cup\{j\},\left(\varphi_{i}\right)_{i \in M \cup\{j\}}, \psi\right)$ would be an element of $\mathcal{C}$ strictly greater than $\left(M,\left(\varphi_{i}\right)_{i \in M}, \varphi\right)$, which is a contradiction.

Finally; as in the proof of Lemma 1 of Section 2, we find $\pi \in \vartheta(\mu)$ extending $\varphi$

Remark 2. It follows from the proof of Theorem 2 that the singleton $i_{0} \in I$ can be replaced by any subset of $I$. More precisely, if $J \subseteq I, \varphi_{i} \in \vartheta\left(\mu_{i}\right)$ for every $i \in J$ and $\varphi \in \vartheta\left(\otimes_{\left.i \in J \mu_{i}\right)}\right.$ such that $\varphi=\otimes_{i \in J \varphi_{i}}$, then there exist $\varphi_{i} \in \vartheta\left(\mu_{i}\right)$ for every $i \in I \backslash J$ and $\pi \in \vartheta(\mu)$ such that $\pi=\otimes_{i \in I} \varphi_{i}$. 
Remark 3. The product-density theorem might raise the following question. Given probability spaces $\left(\Omega_{i}, \Sigma_{i}, \mu_{i}\right)$ and densities $\varphi_{i} \in \vartheta\left(\mu_{i}\right) \quad(i \in I \neq \emptyset)$, does there always exist a $\varphi \in \vartheta(\mu)$ such that $\varphi=\otimes_{i \in I} \varphi_{i}$ holds true if $(\Omega, \Sigma, \mu)$ is a probability space such that

$$
\Omega=\prod_{i \in I} \Omega_{i}, \quad \bigotimes_{i \in I} \Sigma_{i} \subseteq \Sigma, \quad \mu \mid \bigotimes_{i \in I} \Sigma_{i}=\bigotimes_{i \in I} \mu_{i} ?
$$

Consider the product space $\left(\prod_{i \in I} \Omega_{i}, \otimes_{i \in I} \Sigma_{i}, \bigotimes_{i \in I} \mu_{i}\right)$, where $I$ is a non-empty index set, $\Omega_{i}$ is a compact extremally disconnected space (i.e. $\Omega_{i}$ is compact and the closure $\bar{G}$ of an open subset $G$ of $\Omega_{i}$ is open), $\Sigma_{i}=\hat{\mathcal{B}}\left(X_{i}\right), \mu_{i}$ is a diffuse (i.e. without atoms) probability measure, and $\mu_{i}(\bar{G})=\mu_{i}(G)$ for all $G \in \mathcal{T}_{i}, \mathcal{T}_{i}$ the topology of $\Omega_{i} \quad(i \in I)$. Such spaces are given by the hyperstonian space derived from a diffuse probability space, e.g. the hyperstonian space of the Lebesgue measure space on $[0,1]$ will do (see [3]). It is well known that such spaces have a unique strong lifting $\sigma_{i} \in \Lambda\left(\mu_{i}\right)$.

Assume that there exists a product density $\varphi_{0}=\otimes_{i \in I} \sigma_{i} \in \vartheta\left(\otimes_{i \in I} \mu_{i}\right)$. Then the $\operatorname{map} \varphi: \hat{\otimes}_{i \in I} \Sigma_{i} \rightarrow \hat{\otimes}_{i \in I} \Sigma_{i}$, defined by $\varphi(A \triangle N)=\varphi_{0}(A)$ for any $A \in \otimes_{i \in I} \Sigma_{i}$ and $N \subseteq N_{1}$ with $\hat{\otimes}_{i \in I} \mu_{i}\left(N_{1}\right)=0$, is clearly a density and $\varphi=\otimes_{i \in I} \sigma_{i} \in \vartheta\left(\hat{\otimes}_{i \in I} \mu_{i}\right)$.

The canonical projections $f_{i}$ from $\prod_{i \in I} \Omega_{i}$ onto $\Omega_{i}$ are $\left(\mathcal{T}_{\varphi}^{*}-\mathcal{T}_{\sigma_{i}}^{*}\right)$-continuous, where $\mathcal{T}_{\varphi}^{*}$ and $\mathcal{T}_{\sigma_{i}}^{*}$ are the density topologies for $\varphi$ and $\sigma_{i}$, respectively (see [7: Chapter $\mathrm{V}$, Section 1] for the definition). In fact for any $A \in \mathcal{T}_{\sigma_{i}^{*}}^{*}$ it holds true that $A \subseteq \sigma_{i}(A)$ hence

$$
f_{i}^{-1}(A) \subseteq f_{i}^{-1}\left(\sigma_{i}(A)\right)=\varphi\left(f_{i}^{-1}(A)\right), \quad \text { i.e. } f_{i}^{-1}(A) \in \mathcal{T}_{\varphi}^{*} \quad \text { for each } i \in I .
$$

Therefore for the product topology $\prod_{i \in I} \mathcal{T}_{i}$ we get $\prod_{i \in I} \mathcal{T}_{i} \subseteq \mathcal{T}_{\varphi}^{*} \subseteq \dot{\otimes}_{i \in I} \Sigma_{i}$, which is a contradiction according to [3].

So the answer to the above question is to the negative (even if all the $\sigma_{i}$ are strong liftings) and this raises the question "under which conditions can more than one of the $\varphi_{i} \in \vartheta\left(\mu_{i}\right)$ be pre-assigned, in particular, when can all of them be pre-assigned".

Given a compact group $G$ of uncountable weight $w(G)=\alpha$ there exists by [5: Lemma 2.2] a family $\left(H_{\gamma}\right)_{\gamma<\alpha}$ of closed normal subgroups of $G$ with $\bigcap_{\gamma<\alpha} H_{\gamma}=\{e\}, e$ the unit element of $G$, such that for every $\gamma<\alpha$

(i) $H_{\delta} \subseteq H_{\gamma}$ for $\gamma \leq \delta \leq \alpha$

(ii) $H_{\gamma} / H_{\gamma+1}$ is Lie

(iii) $H_{\gamma}=\bigcap_{\beta<\gamma} H_{\beta}$ if $\gamma$ is a limit ordinal.

It follows from the above result that $X_{\gamma}:=H_{\gamma} / H_{\gamma+1}=\left(G / H_{\gamma+1}\right) /\left(G / H_{\gamma}\right)$ for $\gamma>0$ and $X_{0}:=G / H_{1}$ are Lie (hence metrizable) compact groups.

Theorem 3. Let $G,\left(H_{\gamma}\right)_{\gamma<\alpha}$ and $\left(X_{\gamma}\right)_{\gamma<\alpha}$ be as above. Then the following statements are true:

(i) For any Baire probability measure $\mu$ on $G$ there exist a self-consistent family of densities $\varphi_{\gamma} \in \vartheta\left(\mu_{\gamma}\right)$, where $\mu_{\gamma}:=\mu \circ f_{\gamma}^{-1}, f_{\gamma}$ the canonical projection from $G$ onto $G / H_{\gamma} \quad(\gamma<\alpha)$ and $a \varphi \in \vartheta(\mu)$ which is a projective limit of $\left(\varphi_{\gamma}\right)_{\gamma<\alpha}$. 
(ii) There exist a family $\left(\nu_{\gamma}\right)_{\gamma<\alpha}$ of Baire (=Borel) probability measures, each $\nu_{\gamma}$ supported on $X_{\gamma}, \psi_{\gamma} \in \vartheta\left(\nu_{\gamma}\right) \quad(\gamma<\alpha)$ and $\psi \in \vartheta(\lambda)$, $\lambda$ the (normalized) Haar measure on $G$ such that $\psi$ can be represented as the product-density $\otimes_{\gamma<\alpha} \psi_{\gamma}$.

Proof. (i) For any limit ordinal $\gamma<\alpha$ the family $\left(G / H_{\beta}, f_{\beta \delta}, \alpha\right)$ where $f_{\beta \delta}$ is the canonical projection from $G / H_{\delta}$ onto $G / H_{\beta}$, is a projective system. There exists a natural continuous homomorphism from $G$ onto $\operatorname{proj}_{\beta<\gamma} \lim G / H_{\beta}$ whose kernel is $\bigcap_{\beta<\gamma} H_{\beta}$ and $\bigcap_{\beta<\gamma} H_{\beta}=H_{\gamma}$ by relation (iii) preceeding Theorem 3 . So there exists a continuous algebraic isomorphism of the compact group $G / H_{\gamma}$ onto $\operatorname{proj}_{\beta<\gamma} \lim G / H_{\beta}$ which must therefore be a homeomorphism. Thus

$$
G / H_{\gamma}=\operatorname{proj}_{\beta<\gamma} \lim G / H_{\beta}
$$

(cf. [2: Proof of Lemma 9]). Now applying relation (i) preceeding Theorem 2 we get

$$
G=G /\{e\}=\operatorname{proj}_{\gamma<\alpha} \lim G / H_{\gamma} .
$$

But then according to [1: Theorem 2.3] we have

$$
\left(G, \mathcal{B}_{o}(G), \mu\right)=\operatorname{proj}_{\gamma<\alpha} \lim \left(G / H_{\gamma}, \mathcal{B}_{o}\left(G / H_{\gamma}\right), \mu_{\gamma}\right)
$$

and, for any limit ordinal $\gamma<\alpha$,

$$
\left(G / H_{\gamma}, \mathcal{B}_{o}\left(G / H_{\gamma}\right), \dot{\mu_{\gamma}}\right)=\operatorname{proj}_{\beta<\gamma} \lim \left(G / H_{\beta}, \mathcal{B}_{o}\left(G / H_{\beta}\right), \mu_{\beta}\right) .
$$

Hence all the assumptions of Proposition 1 of Section 2 are fulfilled and so there exist a self-consistent family of densities $\varphi_{\gamma} \in \vartheta\left(\mu_{\gamma}\right) \quad(\gamma<\alpha)$ and a $\varphi \in \vartheta(\mu)$ which is a projective limit of $\left(\varphi_{\gamma}\right)_{\gamma<\alpha}$.

(ii) By [5: Theorem 2.3] there exist a family $\left(\nu_{\gamma}\right)_{\gamma<\alpha}$ of Baire (= Borel) probability measures, each $\nu_{\gamma}$ supported on $X_{\gamma}$, and a Baire isomorphism $q_{G}$ from $X_{G}:=\prod_{\gamma<\alpha} X_{\gamma}$ onto $G$ such that $\lambda_{G} \circ q_{G}^{-1}=\lambda$ where $\lambda_{G}:=\otimes_{\gamma<\alpha} \nu_{\gamma}$. By Theorem 2 we find $\psi_{\gamma} \in \vartheta\left(\nu_{\gamma}\right)$ and a product-densitity $\psi_{*} \in \vartheta\left(\lambda_{G}\right)$. Therefore the density $\psi:=\psi_{*} \circ q_{G}^{-1} \in \vartheta(\lambda)$ is the desired one.

Acknowledgement. We are very indebted to the referees for improvements of the exposition and of the proofs of this paper.

\section{References}

[1] Choksi, J. R.: Inverse limits of measure spaces. Proc. London Math. Soc. (3) 8 (1958), $321-342$.

[2] Choksi, J. R.: Measurable transformations on compact groups. Trans. Amer. Math. Soc. 184 (1973), $101 \cdot 124$.

[3] Fremlin, D. H.: Products of Radon measures: a counterexample. Canadian Math. Bull. 19 (1976), $285-289$.

[4] Graf, S. and H. von Weizsäcker: On the existence of lower densities in noncomplete measure spaces. Lect. Notes Math. 541 (1976), 155 - 158. 
[5] Grekas, S.: Structural properties of compact groups with measure-theoretic applications. Israel J. Math. 87.(1994), 89 - 95.

[6] Halmos, P. R.: Measure Theory. New York: Van Nostrand 1950.

[7] Ionescu Tulcea, A. and C. Ionescu Tulcea: Topics in the Theory of Lifting. Berlin. Heidelberg-New York: Springer - Verlag 1969.

[8] Macheras, N. D. and W. Strauss: On various strong lifting properties for topological measure spaces. Suppl. Rend. Cir. Mat. Palermo 28 (1992) (Serie II), 149 - 162.

[9] Macheras, N. D. and W. Strauss: On strong liftings for projective limits. Fund. Math. 144 (1994), $209-229$.

[10] Macheras, N. D. and W. Strauss: On products of almost strong liftings. J. Austral. Math. Soc. (to appear).

[11] Macheras, N. D. and W. Strauss: The product-lifting for complete probability spaces. Math. Proc. Camb. Phil. Soc. (submitted).

[12] Maharam, D.: On a theorem of von Neumann. Proc. Amer. Math. Soc. 9 (1958), 987 994.

[13] Montgomery, D. and L. Zippin: Topological transformation groups. New York: Intersci. Publ., Inc. 1955.

[14] Musial, K.: Projective limits of perfect measure spaces. Fund. Math. 40 (1980), 163 189.

[15] Shelah, S.: Lifting problem of the measure algebra. Israel J. Math. 45 (1983), 90 - 96.

[16] Traynor, T.: An elementary proof of the lifting theorem. Pacific J. Math. 53 (1) (1974), $267-272$. 